\title{
The Impact of Temperature and Relative Humidity on Spatiotemporal Patterns of Infant Bronchiolitis Epidemics in the Contiguous United States
}

Chantel Sloan ${ }^{1 *}$, Matthew Heaton ${ }^{1}$, Sorah Kang ${ }^{1}$, Candace Berrett ${ }^{1}$, Pingsheng $\mathrm{Wu}^{2}$, Tebeb Gebretsadik $^{2}$, Nicholas Sicignano ${ }^{3,4}$, Amber Evans $^{4}$, Rees Lee $^{5}$, Tina Hartert ${ }^{2}$

${ }^{1}$ Brigham Young University, Provo, Utah, USA

${ }^{2}$ Vanderbilt University Medical Center, Nashville, Tennessee, USA

${ }^{3}$ Health ResearchTx, Trevose, PA

${ }^{4}$ Navy and Marine Corps Public Health Center, Portsmouth, Virginia, USA

${ }^{5}$ Naval Medical Research Unit Dayton, Wright Patterson AFB, Ohio, USA

*Correspondence to: Brigham Young University, Life Sciences Building 2048. Tel.: +801 422 3959. chantel.sloan@byu.edu

\begin{abstract}
Infant bronchiolitis is primarily due to infection by respiratory syncytial virus (RSV), which is highly seasonal. The goal of the study is to understand how circulation of RSV is impacted by fluctuations in temperature and humidity in order to inform prevention efforts. Using data from the Military Health System (MHS) Data Repository (MDR), we calculated rates of infant bronchiolitis for the contiguous US from July 2004 to June 2013. Monthly temperature and relative humidity were extracted from the National Climate Data Center. Using a spatiotemporal generalized linear model for binomial data, we estimated bronchiolitis rates and the effects of temperature and relative humidity while allowing them to vary over location and time. Our results indicate a seasonal pattern that begins in the Southeast during November or December,
\end{abstract}


then spreading in a Northwest direction. The relationships of temperature and humidity were spatially heterogeneous, and we find that climate can partially account for early onset or longer epidemic duration. Small changes in climate may be associated with larger fluctuations in epidemic duration.

\section{Keywords}

RSV; climate; respiratory; seasonal

\section{INTRODUCTION}

Bronchiolitis is a lower respiratory tract infection (LRTI) that is predominantly caused by respiratory syncytial virus (RSV) among infants during the winter virus season (McNamara and Smyth 2002). RSV is responsible for between 50 and $80 \%$ of bronchiolitis in infants. An estimated $2.3 \%$ of neonatal (age $0-27$ days) deaths and $6.7 \%$ post-neonatal (age $28-364$ days) deaths were due to RSV related lower respiratory infections (Lozano et al. 2013). For children aged 5 and younger, an estimated 3.4 million hospitalizations per year are related to RSV worldwide, including between 66,000 and 199,000 deaths (Nair et al. 2010). Additionally, infant bronchiolitis has been shown to have a strong causal link with the later development of childhood asthma (Heymann et al. 2004; Wu et al. 2008). Despite the high prevalence and the significant morbidity and mortality, treatment for bronchiolitis is limited to supportive care, and an effective vaccine has not yet been developed. Monthly administration of immunoprophylaxis is the only available preventive measure. However, immunoprophylaxis is very expensive, with expected costs during one RSV season between $\$ 9,939$ and $\$ 13,500$ for children less than two years old (Hampp et al. 2011). Thus understanding factors which impact viral spread is another option for pursuing targeted infection prevention. 
RSV circulation follows distinct annual patterns similar to influenza, peaking primarily between November and March in the northern hemisphere and during April and September in the southern hemisphere (Noyola and Mandeville 2008; Taylor et al. 2016; Walton et al. 2010; Welliver Sr 2007). In the tropics, RSV circulation typically corresponds with the rainy season, though the seasonal peak is not pronounced (Chan et al. 2002). In North America, the annual epidemic is typically found earliest in the southern coastal areas in September and October and then is thought to proceed generally westward and northward (Control and Prevention 2013; Pitzer et al. 2015; Stensballe et al. 2003). A national study by the Centers for Disease Control and Prevention indicated that the peak timing of RSV epidemics can range between December 3rd and March 17th, depending on location (with seasons ending as late as May 5th) (Control and Prevention 2013).

The reasons for these patterns, potential for year-specific fluctuations in RSV epidemics, and how they correspond with the timing of location-specific rates of immunoprophylaxis administration are unknown. For instance, models suggest that climate and demographic factors influence RSV transmission and host susceptibility (Bradley et al. 2005; Gürkan et al. 2000; Welliver Sr 2007; Yusuf et al. 2007). The magnitude of the association between factors that influence RSV dynamics and ecology likely varies across the United States. This is evident as studies of northern climates suggest that temperature is highly correlated with bronchiolitis and RSV rates. However more moderate, coastal climates in the US also experience peaks of bronchiolitis in winter. Even within a single US state, epidemic curves can considerably between cities where climates are very similar (Sloan et al. 2013). While immunoprophylaxis is typically administered between November and March, local rates and timing of administration also vary by location. 
In order to facilitate accurate prediction of bronchiolitis rates as a necessary precursor to more effective prevention strategies, variation across the United States must be better understood. While the ubiquitous annual circulation of RSV is difficult to measure in infant populations across large geographic areas, it is possible to track bronchiolitis in infants using medical claims data. In this study, we performed the largest and most geographically diverse study of temperature, climate and infant bronchiolitis rates conducted to date. The goal of this study was to model variation in spatiotemporal patterns of infant bronchiolitis rates in a nationwide health care database in the United States, and to understand the influence of temperature and humidity on those rates.

\section{METHODS}

\section{Database/Population}

Infants covered by the Department of Defense (DoD) Military Health System (MHS) and whose data are available in the MHS Data Repository (MDR) were studied. The MDR contains data for the entire MHS, which serves all active duty and retired military personnel and their dependents. The MDR contains data on almost 10 million covered individuals (including over 2,300 births per week) and incorporates all the administrative and pharmacy data derived from visits to military medical facilities as well as any civilian network visits billed to the Department of Defense. Approximately $40 \%$ of the births and outpatient visits and $25 \%$ of the inpatient visits occur in military hospitals and clinics with the balance of care being provided by civilian facilities. Covered individuals live across the United States and are demographically representative of the US population as a whole. We used medical codes for diagnoses and 
location data for all infant bronchiolitis events (cases) and location data for all other infants (controls).

Inclusion criteria were infants born in the contiguous United States between January 1, 2003 and June 30, 2013 that were enrolled in the MHS between birth and 1 year of age. Cases were defined as infants diagnosed with bronchiolitis (ICD-9 codes: 466.11, 466.19, 480.1, 0.79.6). Note that 480.1 is a code that refers to pneumonia due to RSV, not bronchiolitis, but which is included as it is a lower respiratory infection due to RSV. We retrieved necessary medical claims and location data for all infant bronchiolitis events (cases) and location data for all other infants (controls). Due to the sensitive nature of these data, residence locations were jittered and birthdates and healthcare visits were skewed to protect patient's privacy. Confidentiality rules prevent disclosing the actual distances and number of days.

The study was approved by the Institutional Review Board (IRB) of both the Naval Medical Center Portsmouth and Brigham Young University.

\section{Grid Cell Aggregation}

For purposes of statistical analysis, case and control counts were aggregated to the 5,396 regularly spaced, spatial grid cells shown in Supplemental Figure 1 at the monthly time scale. Each grid cell in Supplemental Figure 1 is approximately 17 miles $(27 \mathrm{~km})$ in the east-west direction and 30 miles $(48 \mathrm{~km})$ in the north-south direction. For controls, we only have data on infants born from January 1, 2003 forward. Therefore, we don't have a good value for the number of control infants less than one year old until January 2004 (i.e. we use birth data in 2003 to calculate the number of controls for 2004). For this analysis, we consider the number of 
bronchiolitis cases and controls that fall in each of the 5396 grid cells for each month beginning July 1, 2004 to June 30,2013 resulting in $5396 \times 12 \times 9=582,768$ correlated data points.

The spatial grid shown in Supplemental Figure 1 was chosen because the spatial resolution of 5396 grid cells is sufficiently coarse to nullify the spatial uncertainty associated with the jittering of the spatial locations in the data. In other words, given the jittering distance for each location, we are able to locate, within a probability of 0.0001 , the grid cell in which the case (or control) lies. Additionally, this spatial resolution is sufficiently high for purposes of this study, while still being small enough to facilitate a computationally reasonable spatiotemporal analysis (for details, see the Statistical Analysis section below).

One month was chosen as a sufficiently high temporal resolution to produce scientifically interesting results while still coarse enough to prevent the data from being too sparse to calculate rates. A smaller temporal resolution on the order of two weeks would produce case counts as low as 30 for the entire contiguous US, which would jeopardize de-identification.

\section{Predictors: Temperature and Humidity Data}

As a key focus of this study was the impact of climate on bronchiolitis rates, we utilized monthly average temperature data from the National Climatic Data Center (NCDC). The temperature data consists of 7,945,096 daily average temperatures at different land stations throughout the United States, over the study period. For relative humidity, we have 73,705 observations, also gathered at different land stations over the same period of time.

For each month considered in this study, we first calculated the mean daily average temperature (or relative humidity) at each land station then kriged (spatially predicted) these observed monthly averages to the 5396 centroids in the spatial grid shown in Supplemental 
Figure 1. We then calculated the maximum likelihood estimates (MLEs) of a stationary

Gaussian process model using a constant mean and the exponential covariance function then we used the calculated MLEs to perform kriging to the spatial locations.

\section{Statistical Analysis}

To relate temperature and relative humidity to bronchiolitis counts, we used a binomial logistic regression model with spatially and temporally varying random effects. With this approach, the primary parameter of interest (outcome or response variable) is the probability of bronchiolitis at specific space-time locations. This predicted conditional probability can be directly transformed into a bronchiolitis rates by simply multiplying by the desired denominator (e.g. multiplying a probability by 100,000 would give the rate per 100,000 people). Due to this direct relationship, we will primarily use the term probability for the remainder of this paper.

Incorporating space-time random effects into the logistic regression model allows for (i) different areas of the US to have different probabilities of bronchiolitis at different times and (ii) year-to-year variation on the seasonal cycle of bronchiolitis. In other words, for every month of each year, all locations are allowed to have their own probability (or rate) of bronchiolitis. This model captures the spatial and temporal trends of bronchiolitis that we would expect. For example, the probability of bronchiolitis could be high for the Southeast in December 2012 (indicating an outbreak) while, simultaneously, low in the Northwest in December 2012.

We assume the probability (rate) of bronchiolitis at a given location, month and year is dependent upon temperature and relative humidity through a location and month specific effect. Constraining the effect of temperature and relative humidity to vary by location and month (but not by year) allows us to investigate the effect of, e.g., a comparatively cold January (for a 
specific location) on the probability of bronchiolitis (refer to the section Impacts of Climate on Bronchiolitis for an example).

Due to the complexity of our model, we smooth the spatiotemporal random effects as well as the effects of temperature and relative humidity using basis function expansions (Cressie and Johannesson 2008; Higdon 2002; Sun et al. 2012). This allows for easier calculation in fitting our statistical model. Rigorous details for the statistical model used herein are provided below.

\section{Description of Statistical Models}

Let $X_{g m y}$ and $N_{g m y}$ represent the number of bronchiolitis cases and controls, respectively, in spatial grid cell $g$ during month $m$ in year $y$ where $g=1, \ldots, 5396$ and $m \in$ $\{J a n, F e b, \ldots, D e c\}$ and $y=2004, \ldots, 2013$. For this analysis we assume that $X_{g m y}$ follows a binomial distribution with $N_{g m y}$ trials and "success" probability $\theta_{g m y}$ (denoted $\left.X_{g m y} \sim \operatorname{Bin}\left(N_{g m y}, \theta_{g m y}\right)\right)$. In this model, $\theta_{g m y} \in(0,1)$ represents the probability that an infant aged less than 1 year contracts bronchiolitis and is the primary parameter of interest. We emphasize that $\theta_{g m y}$ is allowed to vary over space $(g)$ and time $(m, y)$; in other words, $\theta_{g m y}$ is a random effect. This allows for different areas of the US to have different rates of bronchiolitis at different times. Additionally, the dependence on year $y$ allows for outbreaks to happen at different times in different years. For example, $\theta_{g m y}$ could be high for the Southeast in December 2012 (indicating an outbreak) while, simultaneously, low in the Northwest in December 2012. 
Let $T_{g m y}$ and $H_{g m y}$ represent the average daily temperature and humidity for grid cell $g$ in month $m$ and year $y$. We regress $\theta_{g m y}$ on $T_{g m y}$ and $H_{g m y}$ in a logistic regression framework. Specifically, we set,

$\log \left(\theta_{g m y} /\left(1-\theta_{g m y}\right)\right)=\alpha_{g m y}+\beta_{g m} T_{g m}+\gamma_{g m} H_{g m}+\varepsilon_{g m y}$

where $\alpha_{g m y}$ is a space-time varying intercept, $\beta_{g m}$ is the space-month varying effect of temperature, $\gamma_{g m}$ is a space-month varying effect of relative humidity and $\varepsilon_{g m y}$ are independent, mean zero Gaussian distributed random variates with location-specific variances (e.g. $\left.\varepsilon_{g m y} \sim N\left(0, \sigma_{g}^{2}\right)\right)$. The $\varepsilon_{g m y}$ are necessary to capture overdispersion in the data. Note that, according to Figure 1, many areas of the US have small sample sizes (only 1 or 2 cases). In these regions, we expect counts of bronchiolitis to be overdispersed relative to the mean necessitating the use of $\varepsilon_{g m y}$ to model such overdispersion.

According to Equation (1), the intercepts and coefficients vary across space and time. Specifically, the intercept parameters $\left(\alpha_{g m y}\right)$ allow for baseline rates of bronchiolitis to vary over all locations, months and years. In other words, for every month of each year, all locations are allowed to have their own baseline rate, which corresponds to the natural fluxes in spatial and temporal trends of infections and climate that we would expect. In contrast, the effects of temperature $\left(\beta_{g m}\right)$ and humidity $\left(\gamma_{g m}\right)$ are constrained to vary by space and month only. This constraint is to facilitate year-to-year comparisons. That is, by restricting $\beta_{g m}$ and $\gamma_{g m}$ to vary by space and month only, we are able to investigate the effect of, e.g., a comparatively cold January (for a specific location) on the rate of bronchiolitis. The variation among baseline rates of bronchiolitis $\left(\alpha_{g m y}\right)$ over the spatial and temporal domain of interest is potentially due to climatic (as opposed to the weather-related variables of current temperature or humidity which 
we include in the model) or socioeconomic differences among the various regions. The inclusion of such socioeconomic variables into our modeling framework, however, is less appropriate due to the large spatial regions which we consider. However, future extensions of this work could incorporate such variables in an effort to explain the spatio-temporal variability in the baseline rates.

According to Equation (1), there are 582,768 different $\alpha_{g m y}$ parameters and 64,752 total $\beta_{g m}$ and $\gamma_{g m}$ parameters (a total of 712,272), all of which are correlated in space and time. Traditional spatial methods (e.g. Gaussian process models) are not well equipped to handle such a large number of parameters due to computational requirements (Cressie and Johannesson 2008). To overcome these issues, we model $\alpha_{g m y}, \beta_{g m}$, and $\gamma_{g m}$ using convolutions of basis functions (Higdon 2002). More explicitly, we define

$\alpha_{g m y}=\sum_{k=1}^{K} B_{\alpha, k}(g, m, y) \alpha_{k}^{*}$

where $B_{\alpha, k}(g, m, y)$ are spatiotemporal basis functions and $\alpha_{k}^{*}$ are the associated coefficients. This basis function expansion approach allows for the spatiotemporal field $\alpha_{g m y}$ to be determined by a finite number of unknown coefficients and, subsequently, alleviates computational requirements. For this analysis, we let $B_{\alpha, k}(g, m, y)$ be the product of bisquare basis functions (Sun et al. 2012) over space and natural spline bases over time. We determined the number of basis functions by fitting the model in Equation (1) and finding the model with the lowest value for DIC (Spiegelhalter et al. 2002). The basis functions and corresponding coefficients for $\beta_{g m}$ and $\gamma_{g m}$ - namely $B_{\beta, k}(g, m), B_{\gamma, k}(g, m), \beta_{k}^{*}$, and $\gamma_{k}^{*}$ - are similarly defined.

To perform parameters estimation and inference, we adopt the Bayesian paradigm. We assume $\alpha_{k}^{*}$ are a priori independent with mean 0 and standard deviation $\sigma_{\alpha}^{2}$ and, hierarchically, 
assume $\sigma_{\alpha}^{2}$ follows an inverse-gamma distribution with shape and scale 0.1 (a non-informative prior). We use the same general prior structure for the basis function coefficients for $\beta_{g m}$ and $\gamma_{g m}$. Finally, we also assume independent, inverse-gamma prior distributions for each $\sigma_{g}^{2}$ (the variances of the overdispersion parameters).

Estimation for the model in Equation (1) proceeds by sampling all parameters from the posterior distribution. Specifically, we use a Metropolis within Gibbs sampling algorithm where an adaptive Metropolis algorithm is used to sample each $\theta_{g m y}$ then draws of the remaining parameters are sampled directly from their complete conditional distributions (either a Gaussian or inverse-gamma). We followed the suggestions in Jones et al. (2006) and ran the chain until a random sample of parameters all had Monte Carlo standard errors for the posterior median less than 0.01 .

\section{RESULTS}

The study included 1,032,449 infants who lived in the contiguous United States, cared for by the MHS. We identified 135,920 infants with at least one episode of bronchiolitis between July 1, 2004 and June 30, 2013. This corresponds to 13,164 bronchiolitis cases per every 100,000 infants. We removed 3,064 (2.3\%) of these from the analysis due to missing date or location data. There were no identifiable patterns between those with and without these data. We further removed 7,522 cases that lived in Hawaii or Alaska, as the cases in Hawaii and Alaska were concentrated in too geographically dispersed of a pattern to accurately reflect probabilities across space. We had a total of 125,334 cases remaining for analysis.

The infant study population is summarized in Table 1 and Figure 1 . The table gives a demographic summary of the infants with bronchiolitis (cases) and Figure 1 shows the total 
number of case and control counts across the study time period. We used military ranks of the infant's sponsor as a surrogate of socioeconomic status. Pay scales in the military are based on rank. Monthly base pay salaries are as follows: Junior Enlisted $(\$ 1,956.10)$, Senior Enlisted $(\$ 3,297,12)$, Warrant Officer $(\$ 4,097.48)$, Junior Officer $(\$ 4,179.53)$ and Senior Officer $(\$ 7,308.58)$. Unfortunately, race data were not available for most infants in the MDR. Therefore, we could only derive race for $0.16 \%$ of infants $(\mathrm{N}=206)$ and ethnicity on only $0.17 \%(\mathrm{~N}=210)$. Therefore race numbers are not further subdivided here. We emphasize that, according to Figure 1, we observed few bronchiolitis counts in much of the Midwest and Northern Rocky Mountain region of the United States due to low population numbers. Also, the seasonal pattern of bronchiolitis is different across the spatial region necessitating an analysis that takes spatial variation into account.

\section{Bronchiolitis Rates}

The spatial display of the estimation of bronchiolitis probabilities is depicted in Figure 2 averaged across years. White portions of Figure 2 correspond to regions where the uncertainty in the estimate exceeded 0.2. In other words, white regions are highly uncertain areas with sparse number of bronchiolitis cases and/or controls thereby prohibiting precise estimation of the probability. A movie displaying the probabilities is included as part of the supplemental material.

As shown in Figure 1, during September and October bronchiolitis rates are, generally, low with the exception of a few locations (e.g. south eastern Oregon) that are higher primarily due to lone bronchiolitis cases. Beginning in November and December, probabilities start to increase for the Southeast but remain low until January or February for the Northwest. By 
March and April, probabilities (rates) are still higher in some locations in the Northwest while the Southeast has, generally, decreased back to pre-winter levels. While we show only the averages in Figure 2, this annual flow from the Southeast in November and December to the Northwest in January and February was seen for each of the 9 bronchiolitis seasons included in this analysis.

The seasonal southeast-to-northwest cycle is further illustrated in Figure 3 which displays time series plots of the probability of bronchiolitis for 4 locations across the contiguous US. Each of these locations corresponds to areas that have a strong military presence (and, hence, a higher number of observations in the MHS). Comparing Norfolk, VA (United States east coast) to San Diego and Tacoma (United States west coast), note that the western locations experience peaks in bronchiolitis 1-2 months later than the eastern location, Norfolk. Notably, during the peak months in the west (Tacoma and San Diego), the east (Norfolk) is, commonly, experiencing a decline in bronchiolitis rates. Also, the temporal patterns of high rates in Waco, TX (south central United States) are more prolonged than in the other locations as evidenced by the peaks in Waco enveloping the peaks in the other locations.

While the spatial patterns in bronchiolitis were similar each season, the date of onset, the epidemic peak, the duration of the peak, and the termination of the epidemic varied from year to year. Take, as an example, the time at which the estimated bronchiolitis rate exceeded 0.02 in Tacoma Washington. This elevated rate occurred as early as December in 2005 and 2008 (for the 2005-2006 and 2008-2009 seasons) or as late as February in 2010, 2012 and 2013 (for the 2009-2010, 2011-2012 and 2012-2013 seasons). Specifically, the estimated bronchiolitis rate December 2005 (2008) was 42\% (52\%) higher than the December average of the nine studied 
seasons. As further explained in the next subsection, we seek to explain these seasonal variations using climate information.

\section{Impact of Climate on Bronchiolitis}

Our primary research question herein is how climate, if at all, affects the spatiotemporal variability in bronchiolitis rates. Specifically, can differences in weather (e.g. a warm winter) account for differences in the date of onset, peak, and duration of an infant bronchiolitis epidemic? To answer this question, Figures 4 and 5 display the estimated effect of temperature and the estimated effect of relative humidity, respectively. Both Figure 4 and Figure 5 are on the log-odds scale where negative values indicate that as the average monthly temperature, or relative humidity, increases, bronchiolitis rates decrease and positive values indicate rates increase. Alternatively, the values shown in Figures 4 and 5 can be transformed to denote the percentage increase in bronchiolitis rates due to a 1-degree increase in temperature. For example, suppose the estimate of the effect of temperature was 0.6. If the average monthly temperature in that location and month increases by 1 , the relative predicted probability of bronchiolitis would be $\exp \{0.6\}=1.82$ times as large. Yet another interpretation is that an increase of 1-degree in average monthly temperature corresponds to an $82 \%(100 \times$ $(\exp \{0.6\}-1)=82 \%)$ increase in the predicted probability of bronchiolitis.

The effect of temperature and relative humidity varies over time and space, as seen in Figures 4 and 5. Particularly for increases in temperature (or relative humidity) there are regions where bronchiolitis probabilities tend to increase (e.g. eastern Oregon) while other regions tend to decrease (e.g. Utah). Supplemental Figure 2 shows the average temperature in degrees Celsius 
for September 2012 through April 2013, while Supplemental Figure 3 shows the average relative humidity for the same months. The spatial pattern for other years was similar (data not shown).

Temporally, the spatial patterns in the effect of temperature (Figure 4) are spatially consistent but effects tend to be larger during non-peak months (September, October, March and April). During peak months, effects of temperature are more attenuated but still statistically significant. As an example, the estimate for the effect of temperature on bronchiolitis in Tacoma, WA was -0.08 (with an interval estimate of $(-0.17,-0.06)$ ) suggesting that a unit increase in December average temperature in Tacoma, WA, would lead bronchiolitis probabilities that are between $6 \%$ and $15 \%$ smaller.

The effect of relative humidity largely reflects what is seen in maps of the effect of temperature (Figures 4 and 5). Namely, the most pronounced effects seem to be in the West and Northwest with the largest effects happening in off-peak months (September and October). In many areas, in our model, the effect of relative humidity is of the same direction as the effect for temperature. For example, the estimate of the effect of humidity in Tacoma, WA in December was -0.04 (with an interval estimate of $(-0.06,-0.02))$ suggesting that as the average relative humidity in Tacoma in December increases by 1, the probability of bronchiolitis would be between $6 \%$ and $2 \%$ smaller (consistent with the above described example for temperature).

Given the estimated effects of temperature and relative humidity on bronchiolitis probability, can the higher bronchiolitis probabilities in Tacoma during December 2005 and December 2008, mentioned above, be partially attributed to changes in weather? The average temperature and relative humidity in December 2005 and 2008 were both below average for December with temperatures of $38^{\circ} \mathrm{F}\left(2^{\circ}\right.$ degrees below average $)$ and $34^{\circ} \mathrm{F}(6$ degrees below average) and relative humidity of $75 \%$ ( 2 percent below average) and $74 \%$ ( 3 percent below average). If these 
December temperatures and relative humidity were increased to average levels, we would expect the probability of bronchiolitis to be approximately $21 \%$ lower in 2005 and $45 \%$ lower in 2008 . The $21 \%$ decrease is $50 \%$ of the total $42 \%$ higher bronchiolitis probability in December 2005 suggesting that weather had an effect but did not account for all of the increase in rate during that month. In contrast, the $45 \%$ decrease is $86.5 \%$ of the $52 \%$ higher bronchiolitis rate in December 2008 suggesting that weather, potentially, had a large impact on rates that month.

\section{DISCUSSION}

We used a population of infants cared for under the Department of Defense whose data are contained in the MDR to estimate rates and timing of bronchiolitis epidemics for the contiguous US from July 2004 to June 2013 and assess the impact of temperature and relative humidity on these rates. We utilized a spatiotemporal binomial logistic regression model to obtain estimates in regions with few observed bronchiolitis cases or controls. Furthermore, our model specification allowed for the effect of temperature and relative humidity to vary by month and over the spatial domain.

Results from fitting the above model suggested that seasonal patterns of bronchiolitis tend to begin in the Southeast in November and December before the onset in the Northwest in January and February. These results are similar to those shown using surveillance catchment areas and were remarkably consistent over the 10 -year study period(Stensballe et al. 2003). Our calculated probabilities indicate rates of bronchiolitis health care visits between 30 and 40 per 1,000 infants at epidemic peak. The results are comparable to rates found in a recent study which found overall rates of bronchiolitis hospitalization between 50 and 60 per 1,000 infants in the United States (Doucette et al. 2016). These general patterns, however do have exceptions from 
year to year. Using Tacoma, WA (US west coast) as an example, we found that temperature and relative humidity can be partly responsible for higher rates of bronchiolitis from year-to-year. Particularly, for Tacoma, we found that temperature had a larger absolute impact on probability than relative humidity.

In addition, the impact of relative humidity and temperature appeared to have the greatest impact at a point in time at the beginning of each season's bronchiolitis epidemic, suggesting a potentially important role in the emergence of pathogens causing bronchiolitis. The timing of bronchiolitis is consistent with known patterns of RSV seasonality in the US population, as described. Rates begin in the Southeast and generally progress North and then West.

While there are a number of strengths of this study, namely the large and geographically diverse study population, there are important limitations to be considered. This analysis did not consider the impact of pollution or demographics on bronchiolitis rates, nor were etiologies of bronchiolitis available for most infants. Other climactic conditions such as barometric air pressure, precipitation, sunshine, winds, etc., were also not considered. We were not able to take in-home measurements due to the nature of the dataset. Therefore indoor air temperature and humidity are not considered, though they may play a role in disease spread as persons travel from a cold outdoor area to a warm indoor area. Given the short spatial range of pollutants, we hypothesize that the effect of pollution on bronchiolitis rates are more local than is feasibly possible to consider in an analysis of the contiguous US. Further, it is unknown whether differences in rates may be influenced by spatial variation of RSV risk factors such as premature infant birth rates or smoking rates.

As bronchiolitis is most common during infancy, the code for bronchiolitis during this time period is more reliable. However, as bronchiolitis is a clinical diagnosis without a 
confirmatory test, our data is likely to have some variability subject to individual provider variation in the identification of signs and symptoms of, and thus coding of, bronchiolitis. The impact of resulting noise in the data are likely reduced by the large cohort size, that bronchiolitis is common, and that there are few other diseases mistaken for bronchiolitis in this age group. The data should be interpreted as consistent with real-world clinical diagnosis rather than laboratoryconfirmed RSV, and may be subject to variability in coding seen in computer-based patient records more broadly (Hogan and Wagner 1997).

While this study provides useful information in assessing general spatial and temporal patterns of bronchiolitis, as well as the influence of temperature and humidity, questions remain as to the dynamics of the patterns seen in this analysis. Namely, does bronchiolitis spread within nearby locations or from longer distances? Are viruses that cause bronchiolitis present in communities year round and conditions become optimal for its emergence and spread, or is it introduced annually to communities from outside? What factors drive the initiation of RSV, as well as termination of the epidemic? Is it possible to estimate temporal effects of neighboring locations on bronchiolitis rates? Future studies could approach some of these questions using models that account for variation in epidemic rates in surrounding location.

\section{CONCLUSIONS}

In summary, this study demonstrates the seasonality, location and timing of annual infant bronchiolitis epidemics, with initiation in the southeastern US and spread northwestward. The influence of temperature and humidity was most pronounced at the start and end of epidemics, suggesting that they may contribute to important conditions that allow for the emergence and termination of viral pathogens in the community. 
CONFLICTS OF INTERESTS

Tina Hartert is a consultant for Novavax and Regeneron Pharmaceuticals. All other authors declare they have no actual or potential competing financial interests.

\section{ACKNOWLEDGEMENTS}

This study was funded by Health ResearchTx LLC in collaboration with Naval Medical Center Portsmouth and Navy Marine Corps Public Health Center. Drs. Sloan, Heaton and Berrett are supported by NIEHS grant R03ES025295. Additional funding includes K24 AI 77930 (Tina Hartert). The views expressed in this article are those of the authors and do not necessarily reflect the official policy or position of the Department of the Navy, Department of Defense, nor the U.S. Government. Dr. Lee is a military service member. This work was prepared as part of his official duties. Title 17 U.S.C. $§ 105$ provides that 'Copyright protection under this title is not available for any work of the United States Government.' Title 17 U.S.C. $\$ 101$ defines a U.S. Government work as a work prepared by a military service member or employee of the U.S. Government as part of that person's official duties.

\section{REFERENCES}

Bradley JP, Bacharier LB, Bonfiglio J, Schechtman KB, Strunk R, Storch G, et al. 2005. Severity of respiratory syncytial virus bronchiolitis is affected by cigarette smoke exposure and atopy. Pediatrics 115:e7-e14.

Chan P, Chew F, Tan T, Chua K, Hooi P. 2002. Seasonal variation in respiratory syncytial virus chest infection in the tropics. Pediatr Pulmonol 34:47-51. 
Control CfD, Prevention. 2013. Respiratory syncytial virus activity--united states, july 2011january 2013. MMWR Morbidity and mortality weekly report 62:141.

Cressie N, Johannesson G. 2008. Fixed rank kriging for very large spatial data sets. Journal of the Royal Statistical Society: Series B (Statistical Methodology) 70:209-226.

Doucette A, Jiang X, Fryzek J, Coalson J, McLaurin K, Ambrose CS. 2016. Trends in respiratory syncytial virus and bronchiolitis hospitalization rates in high-risk infants in a united states nationally representative database, 1997-2012. PLoS One 11:e0152208.

Gürkan F, Kıral A, Dağl E, Karakoç F. 2000. The effect of passive smoking on the development of respiratory syncytial virus bronchiolitis. Eur J Epidemiol 16:465-468.

Hampp C, Kauf TL, Saidi AS, Winterstein AG. 2011. Cost-effectiveness of respiratory syncytial virus prophylaxis in various indications. Arch Pediatr Adolesc Med:archpediatrics. 2010.2298 v2013.

Heymann PW, Carper HT, Murphy DD, Platts-Mills TA, Patrie J, McLaughlin AP, et al. 2004. Viral infections in relation to age, atopy, and season of admission among children hospitalized for wheezing. J Allergy Clin Immunol 114:239-247.

Higdon D. 2002. Space and space-time modeling using process convolutions. Quantitative methods for current environmental issues 3754 .

Hogan WR, Wagner MM. 1997. Accuracy of data in computer-based patient records. J Am Med Inform Assoc 4:342-355.

Lozano R, Naghavi M, Foreman K, Lim S, Shibuya K, Aboyans V, et al. 2013. Global and regional mortality from 235 causes of death for 20 age groups in 1990 and 2010: A systematic analysis for the global burden of disease study 2010. The Lancet 380:2095-2128. 
McNamara PS, Smyth RL. 2002. The pathogenesis of respiratory syncytial virus disease in childhood. Br Med Bull 61:13-28.

Nair H, Nokes DJ, Gessner BD, Dherani M, Madhi SA, Singleton RJ, et al. 2010. Global burden of acute lower respiratory infections due to respiratory syncytial virus in young children: A systematic review and meta-analysis. The Lancet 375:1545-1555.

Noyola D, Mandeville P. 2008. Effect of climatological factors on respiratory syncytial virus epidemics. Epidemiol Infect 136:1328-1332.

Pitzer VE, Viboud C, Alonso WJ, Wilcox T, Metcalf CJ, Steiner CA, et al. 2015. Environmental drivers of the spatiotemporal dynamics of respiratory syncytial virus in the united states. PLoS Pathog 11:e1004591.

Sloan CD, Gebretsadik T, Wu P, Carroll KN, Mitchel EF, Hartert TV. 2013. Spatiotemporal patterns of infant bronchiolitis in a tennessee medicaid population. Spatial and spatio-temporal epidemiology 6:17-23.

Spiegelhalter DJ, Best NG, Carlin BP, Van Der Linde A. 2002. Bayesian measures of model complexity and fit. Journal of the Royal Statistical Society: Series B (Statistical Methodology) 64:583-639.

Stensballe LG, Devasundaram JK, Simoes EA. 2003. Respiratory syncytial virus epidemics: The ups and downs of a seasonal virus. The Pediatric infectious disease journal 22:S21-S32.

Sun Y, Li B, Genton MG. 2012. Geostatistics for large datasets. In: Advances and challenges in space-time modelling of natural events:Springer, 55-77.

Taylor S, Lopez P, Weckx L, Borja-Tabora C, Ulloa-Gutierrez R, Lazcano-Ponce E, et al. 2016. Respiratory viruses and influenza-like illness: Epidemiology and outcomes in children aged 6 months to 10 years in a multi-country population sample. J Infect. 
Walton N, Poynton M, Gesteland P, Maloney C, Staes C, Facelli J. 2010. Predicting the start week of respiratory syncytial virus outbreaks using real time weather variables. BMC Med Inform Decis Mak 10:68.

Welliver Sr RC. 2007. Temperature, humidity, and ultraviolet b radiation predict community respiratory syncytial virus activity. The Pediatric infectious disease journal 26:S29-S35.

Wu P, Dupont WD, Griffin MR, Carroll KN, Mitchel EF, Gebretsadik T, et al. 2008. Evidence of a causal role of winter virus infection during infancy in early childhood asthma. Am J Respir Crit Care Med 178:1123.

Yusuf S, Piedimonte G, Auais A, Demmler G, Krishnan S, Van Caeseele P, et al. 2007. The relationship of meteorological conditions to the epidemic activity of respiratory syncytial virus. Epidemiol Infect 135:1077-1090.

\section{FIGURE LEGENDS}

Figure 1. (a) Total counts of the number of bronchiolitis cases and (b) controls from the MHSD.
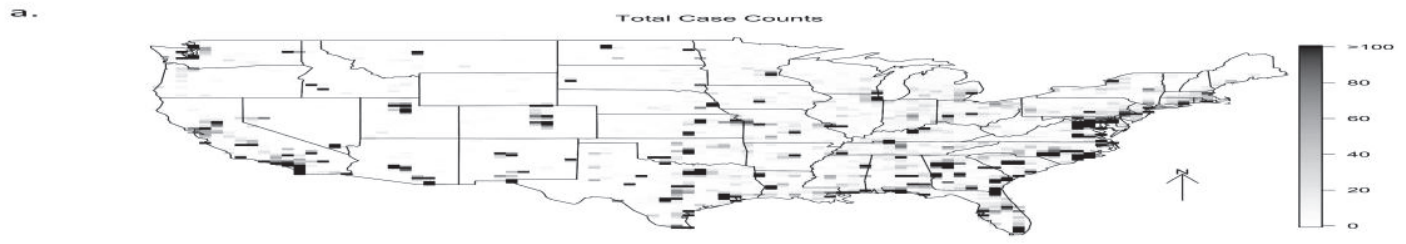

b.

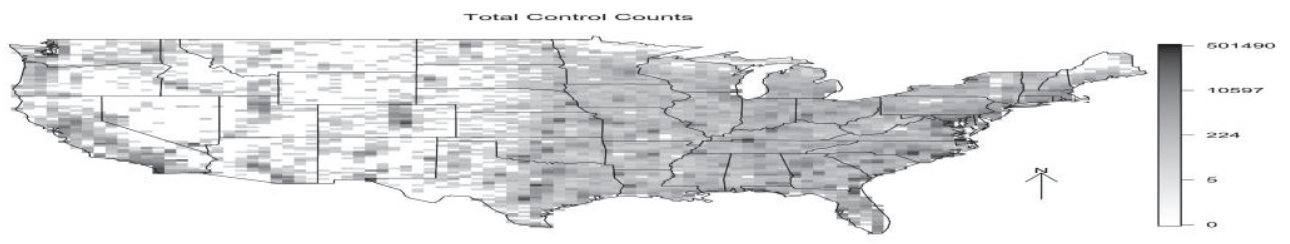


Figure 2. Maps of the estimated probability of bronchiolitis $\left(\theta_{\text {gmy }}\right)$ on the log-odds scale such that values near 0 indicate $\theta_{\text {gmy }}=0.5$ and values near -6 indicate $\theta_{\text {gmy }} \approx 0.002$. Areas in white are those areas with an overly high uncertainty due to too few observations and, hence, were omitted.
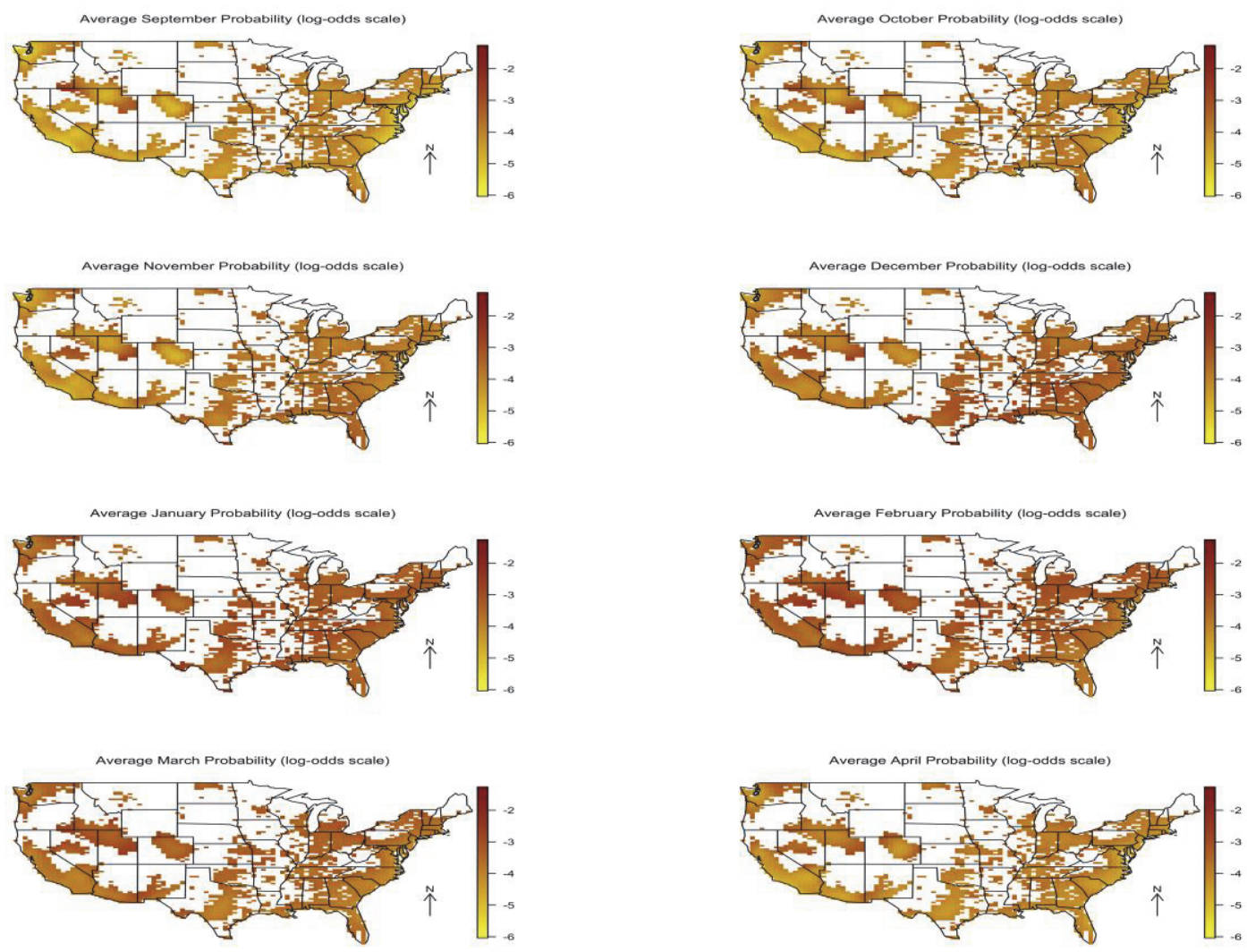

Figure 3. Time series plots of the posterior median for 4 locations across the contiguous US.

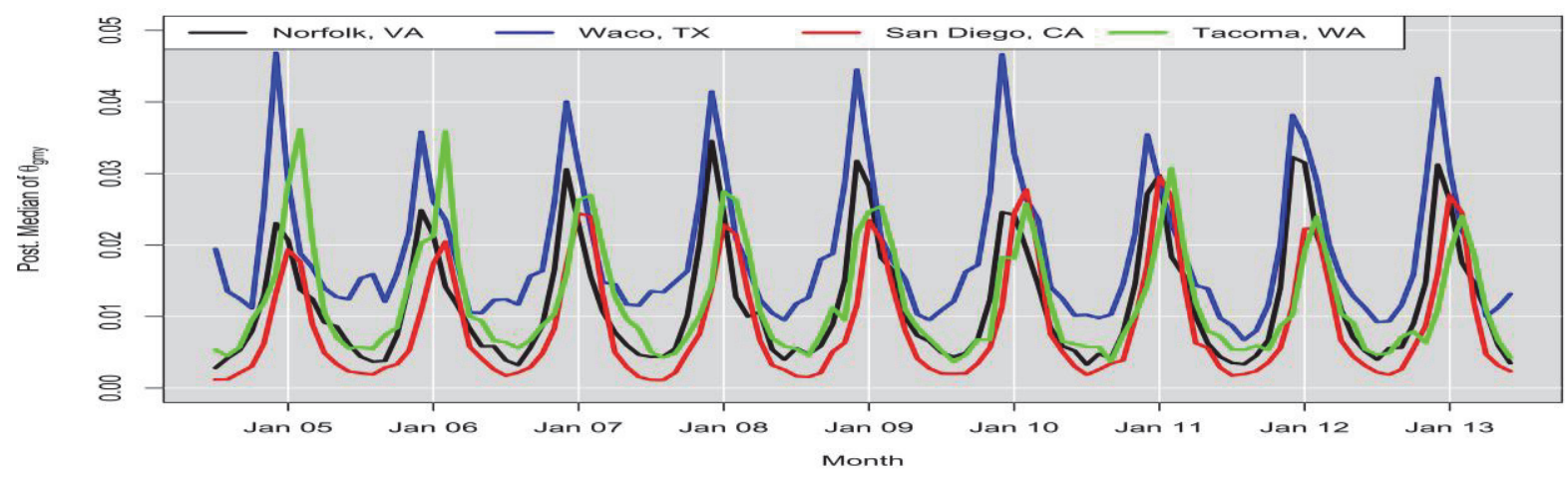


Figure 4. Estimates of the effect of temperature on bronchiolitis $\left(\beta_{g m}\right)$. Positive values indicate that increases in temperature, holding all else constant are associated with increases in the predicted probability of bronchiolitis. Conversely, negative values indicate that increases in temperature, holding all else constant, are associated with decreases in the predicted probability bronchiolitis. Values near zero indicate no association between change in temperature and bronchiolitis.
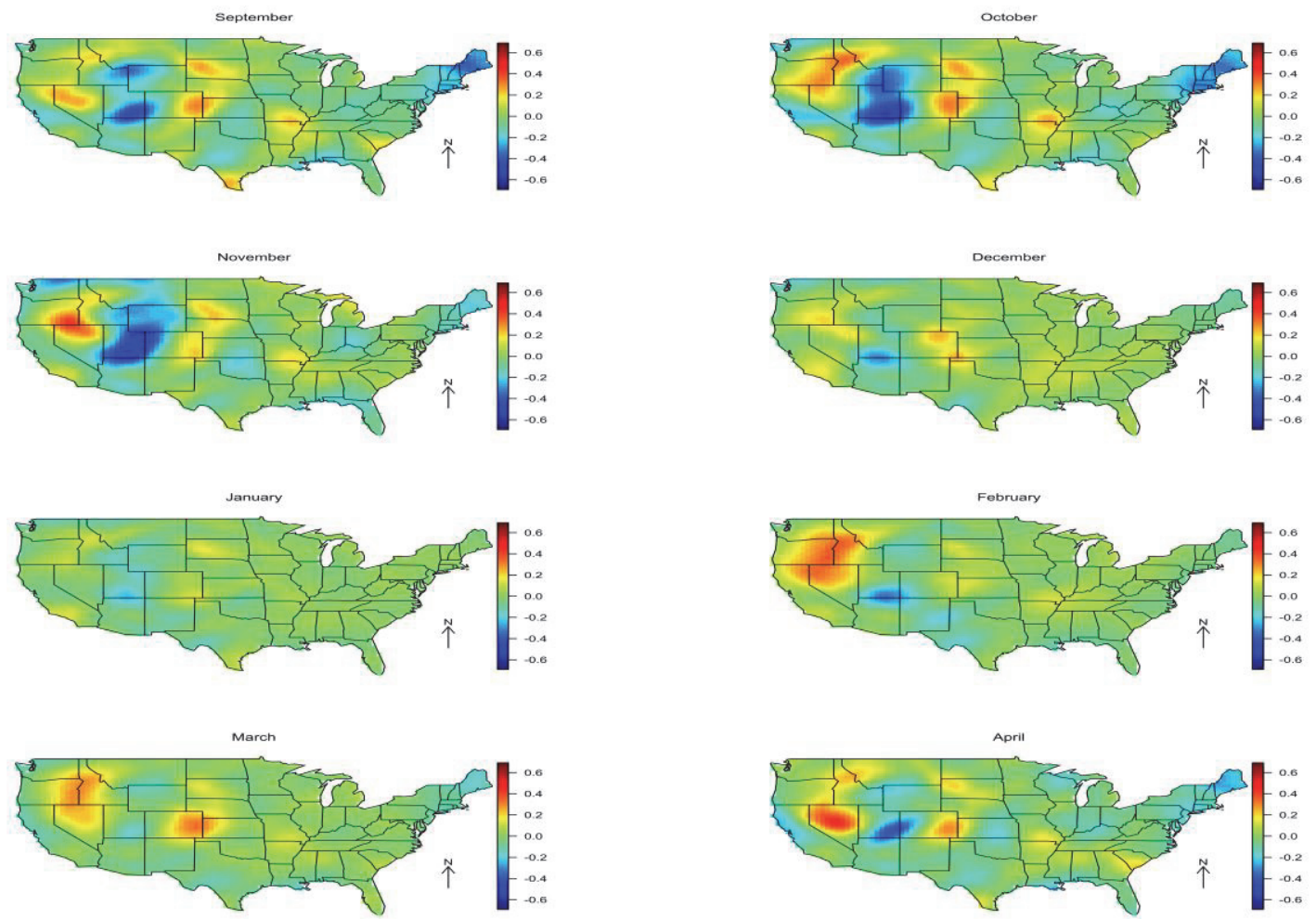

Figure 5. Estimates of the effect of relative humidity on bronchiolitis rates. Negative values indicate a negative relationship while positive values indicate a positive relationship.
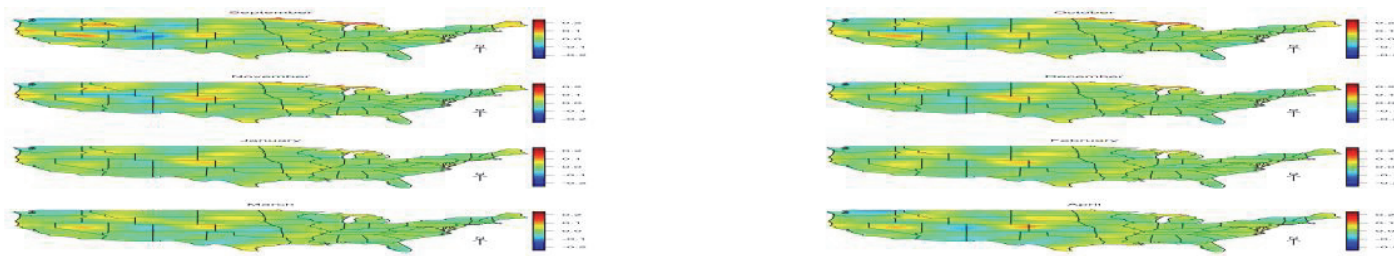


\section{TABLES}

Table 1. Demographic Characteristics. The demographic characteristics for a subset of infants less than 1 year old in the MDR who were the children of active duty officers, and the subset of all infants who had a hospital, emergency department, or outpatient visit for bronchiolitis between 2003 and 2011. Rank of the military sponsor of the infant represents socioeconomic status. Race and ethnicity for infants were not available in the MDR for infants. Race data were available on only $0.16 \%$ of infants $(\mathrm{N}=206)$ and ethnicity on only $0.17 \%(\mathrm{~N}=210)$, therefore race numbers are not further subdivided here. Socioeconomics are based on military pay scale as listed in the table from lowest to highest.

\begin{tabular}{rcc}
\hline & \multicolumn{2}{c}{ Bronchiolitis Cases $(\mathbf{N}=\mathbf{1 2 5 , 3 3 4 )}$} \\
\cline { 2 - 3 } Female & Number & Percent \\
Unknown & 72,850 & 58.1 \\
\hline Demographic Characteristics & 49 & 41.8 \\
Rank of Military Sponsor & & 0.04 \\
Enlisted, Junior & 44,817 & 35.8 \\
Enlisted, Senior & 60,496 & 48.3 \\
Officer, Warrant & 1,413 & 1.1 \\
Officer, Junior & 12,238 & 9.8 \\
Officer, Senior & 6,279 & 5.0 \\
& & \\
Other/Unknown & 91 & 0.07 \\
& & 7.60 \\
Cases per year & 9,520 & 8.70 \\
$\mathbf{2 0 0 3}$ & 10,901 & 9.41 \\
$\mathbf{2 0 0 4}$ & 11,790 & 10.68 \\
$\mathbf{2 0 0 5}$ & 13,389 & 11.61 \\
$\mathbf{2 0 0 6}$ & 14,554 & 12.99 \\
$\mathbf{2 0 0 7}$ & 16,286 & 13.62 \\
$\mathbf{2 0 0 8}$ & 17,072 & 12.77 \\
$\mathbf{2 0 0 9}$ & 15,999 & 12.62 \\
$\mathbf{2 0 1 0}$ & 15,823 & \\
$\mathbf{2 0 1 1}$ & & \\
& &
\end{tabular}

